\title{
Impaired subjective self-control in alcohol use: An ecological momentary assessment study
}

\author{
Danielle Remmerswaal*, Joran Jongerling, Pauline J. Jansen, Charly Eielts, Ingmar H.A. Franken \\ Department of Psychology, Education and Child Studies, Erasmus University Rotterdam, P.O. Box 1738, 3000 DR, Rotterdam, the Netherlands
}

\section{A R T I C L E I N F O}

\section{Keywords:}

Alcohol

Subjective control

Craving

Ecological momentary assessment

\begin{abstract}
A B S T R A C T
Background: While both theory and empirical findings have supported impaired self-control as a crucial factor in understanding problem drinking, little is known about the relationship of self-control and drinking in naturalistic settings. The present study uses Ecological Momentary Assessment (EMA) to examine the predictive relationships between impaired subjective self-control, craving and alcohol use in everyday life.

Methods: A sample of 172 regular drinkers responded on their smartphone to three random prompts each day for seven days in which amount of perceived self-control and craving were measured with self-report. In the meantime, participants were instructed to initiate an EMA report when they started drinking alcohol.

Results: Findings supported the hypotheses that impaired self-control and higher craving levels were prospectively related to the likelihood that people will drink. That is, on random assessments that preceded drinking (i.e., were within two hours of drinking), perceived self-control was lower and craving was higher compared to random assessments that were not followed by drinking. Additionally, during drink consumption, impaired selfcontrol and craving were associated with a higher amount of expected alcohol consumption. Findings further indicated that subjective self-control acted as a moderator of the relationship between craving and alcohol consumption during drinking occasions.

Conclusions: By using a smartphone mobile application, this study showed that impaired subjective self-control and craving are prospectively related to alcohol use in the real-world. Furthermore, findings are consistent with theories of addiction that substance use might be associated with the interplay of control processes and increased motivation.
\end{abstract}

\section{Introduction}

The concept of impaired self-control has been viewed as a central feature of addiction according to several theoretical models of substance use and substance use disorders (Leeman et al., 2014; Goldstein and Volkow, 2002). Jellinek (1960) was one of the first researchers to define the term 'loss of control' as the inability of alcohol dependent patients to stop drinking once drinking has begun, resulting in a binge drinking episode. To reflect the current notion that diminished control is seen to varying degrees in the general population, the concept of loss of control is since then replaced by less extreme views like 'impaired' self-control. Self-control refers to the ability to exert control over habitual or dominant responses in order to advance distal motives over momentarily proximal motives (Baumeister et al., 2007; Duckworth et al., 2016; Fujita, 2011). Exerting self-control over impulses that are activated by salient temptations requires cognitive processes known as "executive functions" (Baumeister, 2002; Bickel et al., 2012), which are a collection of separate but related functions involved in goal-directed behaviour. The three core components of executive functioning are inhibitory control, working memory, and mental set shifting (Miyake et al., 2000). Impaired control over alcohol use specifically refers to a breakdown of an intention to limit drinking (Heather et al., 1993), suggesting that executive functioning is suboptimal. A recent application of this concept can be found in two of the substance use disorder criteria of the Diagnostic and Statistical Manual of mental disorders (DSM-5): "using alcohol more or longer than intended" and, "persistent desire or repeated unsuccessful efforts to quit or cut down drinking" (American Psychiatric Association, 2013).

There is a wealth of research supporting the role of control processes in the etiology of alcohol use disorder. One core component of executive functioning that is important in self-control is inhibitory control. Inhibitory control can be defined as the ability to delay, stop or change a behavioral response, and is typically measured by the Go/NoGo and the Stop Signal tasks (Logan et al., 1997). It has been suggested that

\footnotetext{
* Corresponding author.

E-mail address: remmerswaal@essb.eur.nl (D. Remmerswaal).
} 
poor inhibitory control measured with these tasks acts as both a consequence and as contributor for drug use (López-Caneda et al., 2014; Verdejo-Garcia et al., 2008; de Wit, 2009). Studies of the acute effects of alcohol have indeed demonstrated that alcohol impairs inhibitory control (Field et al., 2010; Fillmore, 2003). In addition, research suggests that inhibitory control plays a potentially causal role in alcohol abuse. A study by Jones et al. (2011), for example, revealed that experimentally manipulating inhibitory control has a causal effect on alcohol consumption. In addition, inhibitory control is linked to heavier drinking in non-dependent drinkers (Christiansen et al., 2012). Looking at impaired control over alcohol use measured by self-report, retrospective accounts of alcoholic adults show that impaired control is reported as the dependence symptom that develops earliest (Langenbucher and Chung, 1995). This is well in line with the results of a recent network analysis study that found that the most central symptom of alcohol abuse was drinking more alcohol than planned (Rhemtulla et al., 2016). Finally, in a study by Leeman et al. (2009) it was reported that perceived impaired control over alcohol use prospectively predicted alcohol related problems. These findings indicate that impaired control over alcohol use may serve as a first symptom that precipitate other symptoms of alcohol use disorder and gives support for the notion that this is a key concept in the development of problematic drinking.

In summary, both theory and empirical findings have supported impaired control as a crucial factor in understanding problem drinking. Nevertheless, research to date on control over alcohol use has predominantly utilized experimental and longitudinal methodologies, and little is known about proximal relationships between control and alcohol use as it manifests in daily life. This is particularly important since control over alcohol use may not only be a stable trait, but is thought to be a dynamic construct that fluctuates over time influenced by internal and environmental factors (Jones et al., 2013).

Craving is another central characteristic of addiction and refers to the subjective desire or urge to drink (American Psychiatric Association, 2013). Alcohol craving can be elicited by conditioned cues but also by other factors such as fluctuations in mood or opportunities to drink (Robinson and Berridge, 2001; Baker et al., 2004). Historical theoretical accounts suggest that impaired control may (partially) reflect the problem with controlling alcohol use in response to craving (Jellinek, 1960). This is well in line with contemporary theories of addiction that propose that both deficits in control processes and an increased motivation for substance use contribute to the inability to control drug use (Goldstein and Volkow, 2002; Jentsch and Taylor, 1999; Wiers et al., 2007). Following these theories it seems plausible that alcohol use might be affected by the interplay of low levels of perceived control and high levels of subjective craving. Specifically, impaired control may be a moderator of the impact of craving on alcohol use. That is, individuals scoring higher on impaired control may be more influenced by the effects of craving. Supporting this line of reasoning, Wardell et al. (2015) investigated control by craving effects in a sample of young heavy drinkers and concluded that trait impaired control acted as a moderator of the relationship between craving and alcohol self-administration in the laboratory. In another laboratory study it was shown that heavy drinkers with ineffective response inhibition showed enhanced craving in response to alcohol cues (Papachristou et al., 2012). Given the view that impaired control may make it difficult for drinkers to ignore salient alcohol related cues that elicit craving, it is important to study the dynamic interplay between those concepts in real-life.

With the development of mobile technologies, it has become possible to collect real-time data in the natural environment by using, for example, smartphones. One such technology to acquiring real-time data is ecological momentary assessment (EMA; Shiffman et al., 2008). The advantage of EMA methods is that it becomes possible to study behavioral, affective, and situational variables as it occurs over time in the contexts in which it normally occurs. This will enhance accuracy of reporting and it allows researchers to study the dynamic interplay between those variables. The use of momentary assessment is particularly important when the phenomena of interest are subject to momentary changes which is the case for perceived control and subjective craving. EMA methods have already provided rich insights into the temporal unfolding of experiences and events in substance use (see Shiffman, 2009). In addition, studies that used EMA methods demonstrated the role of craving and substance use in real-life (Serre et al., 2015). However, little is known about the role of self-control on drinking in naturalistic settings, outside of the laboratory. To date, only one study investigated associations between inhibitory control and alcohol consumption using EMA methods (Jones et al., 2018b). In this study, they objectively measured inhibitory control in daily life and showed that deterioration in inhibitory control across the day was predictive of amount of alcohol consumption later on that day. The lack of ecologically valid studies investigating the role of control on craving and drinking in real life underscores the need for more research to advance our understanding of these important concepts in alcohol research. Moreover, it is important to investigate these associations in a 'normal' population of drinkers since loss of control over alcohol use is a first indication of problem drinking, and even non-problematic regular drinking can have serious detrimental effects on health (Wood et al., 2018).

The present study uses EMA methods to examine the associations between perceived control over alcohol use, craving, and daily alcohol consumption in the natural environment of regular (at least once a week) drinkers. This study can disclose whether the findings from retrospective and laboratory studies on the role of impaired control over alcohol use generalize to the real-life, and may capture immediate precursors that affect control and alcohol use. For this purpose, participants downloaded an application on their smartphones and responded to random prompts for seven days. In addition, participants were instructed to initiate an assessment when they started drinking alcohol. First of all, it was hypothesized that impaired control and craving are prospectively associated with the likelihood that people will drink. More specifically, craving will be higher and control will be lower on assessments that are proximate of a user-initiated alcohol assessment. Second, we hypothesized that craving will be higher and self-control lower during the user-initiated drinking assessments compared to random assessments, and that increased craving and poorer control predict increased alcohol consumption during a drinking assessment. Finally, it is hypothesized that the relationship between craving and alcohol use during the user-initiated drinking assessments will be moderated by subjective self-control. More specifically, based on theoretical models of addiction, we expected that increased craving would predict drinking outcomes more strongly in individuals with poorer control over alcohol use.

\section{Method}

\subsection{Participants}

A convenience sample was recruited using web-based advertising. Participants were 175 regular drinkers (72 men, 103 women) between ages 18 and $66(M=31.1, S D=13.91)$. Most of the participants were from Dutch descent (94.4\%) and all had a good mastery of the Dutch language. Inclusion criteria were: minimum age of 18 , drinking at least once weekly, and being in the possession of an Android or iOS compatible smartphone. The participants did not receive an incentive for their participation.

\subsection{Procedure}

The LifeData platform (www.lifedatacorp.com) was used to develop the application and securely collect data. The participants were instructed to download the LifeData application on their smartphone and 
to download the relevant study survey package to start participation. After participants had finished a start-up session in which general information was provided and informed consent was obtained, the application alerted participants at three random times between 10 a.m. and 10 p.m. for seven consecutive days to complete a short questionnaire (random assessment; RA). Prompts that were not completed within $90 \mathrm{~min}$ of the notification alert disappeared and were marked as missed. In addition, participants were asked to initiate an assessment when they started drinking alcohol (user-initiated alcohol assessment; AA).

\subsection{Measures start up session}

\subsubsection{Problems with alcohol use}

The validated Alcohol Use Disorders Identification Test (AUDIT; Babor et al., 2001; Dutch translation: Schippers and Broekman, 2010) was employed to measure the severity of alcohol use-related problems. The AUDIT consists of 10 questions that can be divided into three subscales: 1) alcohol consumption (items 1-3), 2) drinking behavior/ dependence (items 4-7), and 3) alcohol-related problems or consequences (8-10). Items 1 to 8 are scored on a 5-point Likert scale ranging from 0 (never) to 4 (almost every day). Items 9 and 10 are scored on a 3-point scale with values 0 (no), 2 (yes, but not in the last year), and 4 (yes, during the last year). The total score ranges from 0 to 40 with a higher score indicating more severe alcohol use-related problems. A score of 8 or more is indicative of harmful alcohol use (Babor et al., 2001). The internal consistency of the AUDIT in the current study was good (Cronbach's alpha $=.79$ ).

\subsubsection{Perceived drinking control}

Perceived drinking control was measured using part three of the Impaired Control Scale (ICS; Heather et al., 1993). Subjects were asked to report on ten items (e.g., I would start to drink, even if I decided not to) which assesses beliefs regarding impaired control over drinking behavior. Items are rated on a 5-point scale ranging from 0 (strongly disagree) to 4 (strongly agree). The ICS has good psychometric properties (Heather et al., 1993), and Cronbach's alpha in this sample was .70.

\subsection{Measures ecological momentary assessment}

\subsubsection{Alcohol use}

During random assessments, participants were asked if they had consumed alcohol since the last assessment (user-initiated or random assessment) and how many standard alcoholic drinks they had consumed. During the user-initiated alcohol assessment, participants were asked the total number of drinks they thought they would consume.

\subsubsection{Craving}

During random and user-initiated assessments, participants rated their urge to drink on an 11-point Likert scale from no urge (0) to strongest ever (100). This is a widely used measure in EMA research.

\subsubsection{Control}

Perceived control was assessed during random and user-initiated assessments with a single-item measure. Prompts stated, "How much control do you feel you have over your drinking?" with end-point anchors no control (0) and a lot of control (100).

Additional items, not reported here, assessed contextual variables, drinking motives, affect and subjective responses to alcohol (Supplementary Methods).

\subsection{Statistical analyses}

Due to the nested structure of our data, with multiple measurements (level 1) nested within individuals (level 2), we first determined for all analyses if multilevel analysis was necessary. This was done by first fitting baseline models to the data that only contained measurement occasion (time) as a predictor, and determining whether there was a significant amount of variance on the second, or individual level. For the analyses where there was indeed significant variance on level 2 and where multilevel analysis was therefore necessary, we analyzed our data using multilevel regression analysis with HLM 7.01. For the moderation analysis, in which we examined whether control acted as a moderator of the relationship between craving and alcohol consumption during a drinking session, multilevel analysis was not necessary, due to the absence of variance on the second level. This analysis was therefore carried out in SPSS version 23 using the PROCESS module by Hayes (2012).

\section{Results}

\subsection{Participant characteristics}

Almost half of the participants (49\%) reported drinking alcohol at least 2-3 times a week, and $88 \%$ reported drinking at least 2-4 times a month as measured by the AUDIT. In addition, the average number of drinks during a drinking session was four or less for $74 \%$ of the participants. Participants scored a mean of $8.51(S D=4.80$, range $1-25)$ on the AUDIT; $50 \%$ of participants scored eight or higher, indicating harmful alcohol use (Babor et al., 1992). In general, participants scored below average on part 3 of the Impaired Control Scale with a mean score of $18.56(S D=2.88)$. Multilevel analysis showed that level of impaired control over alcohol use measured with part 3 of the ICS in the pre-assessment, significantly predicted subjective self-control during the EMA assessments (while controlling for the repeated measures) $(b=-5.40, t(163)=-2.59, p=.011$, beta $=-.15)$. More specifically, higher scores on the ICS were negatively related to self-control in daily life. In addition, multilevel analysis showed that alcohol intake differed between random and user initiated assessments. That is, assessment type significantly predicted alcohol intake $(b=-1.69, t(1982)=-16.22$, $p<.001$ ), indicating that alcohol intake was lower on random assessments compared to user-initiated alcohol assessments. Descriptive information for baseline participant characteristics and EMA assessments are shown in Table 1.

\subsection{Ecological momentary assessment reports and compliance}

We first examined the compliance rate for completing random prompts. The maximum number of possible RAs each day was 3 . As a result, 21 ratings were possible for those who participated for 7 days. Participants completed a total of 1742 random assessments (out of 3675 possible prompts, $47.4 \%$ compliance rate). Multilevel analysis takes missing data into account by including all available information. In case the missing data is random, this leads to unbiased estimates. In

Table 1

Participant Characteristics $(N=175)$.

\begin{tabular}{lll}
\hline Baseline Characteristics & & \\
\hline $\begin{array}{l}\text { Age }(M, S D) \\
\text { Gender (female, male) }\end{array}$ & $31.19(13.91)$ & \\
$\begin{array}{l}\text { Descent (\%) } \\
\text { Dutch }\end{array}$ & 103,72 & \\
Other & $94.2 \%$ & \\
AUDIT (M, SD) & $5.8 \%$ & \\
ICS part 3 (M, SD) & $8.51(4.80)$ & AA \\
EMA measures & $18.56(2.88)$ & $86.98(20.51)$ \\
Control (M, SD) & RA & $42.69(36.33)$ \\
Craving (M, SD) & $92.29(15.84)$ & $2.06(3.49)$ \\
Alcohol use (M, SD) & $17.78(27.75)$ & \\
& $0.19(1.32)$ & \\
\hline
\end{tabular}

Note: AUDIT $=$ Alcohol Use Disorders Identification Test; ICS = Impaired Control Scale; EMA = Ecological Momentary Assessment; RA = random assessments; AA = user-initiated alcohol assessments. 
Table 2

Multilevel analyses examining whether control is lower and craving is higher for random assessments that are within two hours of an alcohol assessment.

\begin{tabular}{|c|c|c|c|c|c|c|}
\hline & \multicolumn{3}{|l|}{ Control } & \multicolumn{3}{|l|}{ Craving } \\
\hline & $b$ & SE & $t$ & $b$ & SE & $t$ \\
\hline Intercept & 91.079 & 1.201 & $75.811^{* *}$ & 14.502 & 1.591 & $8.114^{* *}$ \\
\hline Time & .003 & .007 & .487 & .032 & .014 & $2.343^{*}$ \\
\hline Within two hours & -3.401 & 1.355 & $-2.510^{*}$ & 18.218 & 2.498 & $7.291 * *$ \\
\hline Alcohol use T0 & -1.443 & 0.242 & $-5.963^{* *}$ & 5.796 & .433 & $13.381 * *$ \\
\hline
\end{tabular}

Within two hours = random assessments that are within two hours of an alcohol assessment (1) or are outside that time-frame (0). Alcohol use T0 $=$ the amount of alcohol consumed within two hours before the random assessments. Control: $\mathrm{R}^{2}$ level $1=.17, \mathrm{R}^{2}$ level $2=.18$. Craving: $\mathrm{R}^{2}$ level $1=.35, \mathrm{R}^{2}$ level $2=.09 .{ }^{*} \mathrm{P}$-value $<.05, * * \mathrm{P}$-value $<.001$.

addition, 163 of the participants initiated at least one alcohol assessment (93\%) with 523 AAs in total.

\subsection{Perceived control and craving as prospective predictors of alcohol use}

To test the hypothesis that impaired control and craving are prospectively associated with the likelihood that people will drink, we investigated whether craving was higher and control lower on assessments in the two hours preceding (T0) a self-initiated alcohol assessment (T1) (see Table 2). Both control and craving had significant variance on the second, or individual, level (control: $\sigma_{\text {level } 2}^{2}=161.439$, $p<.001$, ICC $=.56$; craving: $\sigma_{\text {level } 2}^{2}=161.346, p<.001$, ICC $=.23$ ), therefore the hypotheses were tested with two multilevel analyses; one with control, and one with craving as the dependent variable (both located on level 1). Both models included measurement occasion (time: used to account for the repeated measures) and a dummy-variable that indicated whether measurements took place two hours before a userinitiated alcohol measurement or not (Within two hours: $0=$ not within $2 \mathrm{~h}, 1=$ within two hours) as level 1 predictors. In addition, we controlled for previous alcohol consumption (Alcohol use TO) by including the amount of alcohol consumed within two hours before the random assessments. Results of the multilevel model with control as dependent variable and moment of random assessment (within two hours or outside two hours) as the predictor showed that perceived control was lower prior to drinking alcohol $(b=-3.40, t(1427)=-2.51, p=.012)$ than on assessments that were more than two hours before drinking alcohol. In addition, previous alcohol consumption made a unique and significant contribution to control levels. Thus, a lower level of perceived self-control was partly determined by alcohol use on an earlier point-in-time $(b=-1.44, t(1427)=-5.96, p<.001)$. Furthermore, in a similar analysis with craving as dependent variable, we found that craving increased on RAs within two hours of drinking alcohol $(b=18.22, t(1507)=7.29, p<.001)$ as compared to RAs that were outside two hours of an alcohol assessment. Moreover, previous alcohol consumption also significantly predicted higher levels of craving $(b=5.79, t(1507)=13.38, p<.001)$.). In addition, we investigated the relation between subjective self-control and craving stratified by moment of random assessment (not within $2 \mathrm{~h}$ vs within two hours of drinking occasion). Results of the multilevel model with craving as dependent variable and perceived control and moment of random assessment as the predictors showed that perceived control negatively predicted craving $(b=-65, t(1386)=-13.34, p<.001)$. The interaction effect of control and moment of random assessment (within two hours or outside two hours) on craving was marginally significant $(b=-.24, t(1386)=-1.92, p=.056)$. This may indicate that the relationship between craving and perceived control is stronger prior to drinking alcohol.
Table 3

Multilevel analyses comparing perceived control and craving during random assessments versus alcohol assessments.

\begin{tabular}{|c|c|c|c|c|c|c|}
\hline & \multicolumn{3}{|l|}{ Control } & \multicolumn{3}{|l|}{ Craving } \\
\hline & $b$ & SE & $t$ & $b$ & SE & $t$ \\
\hline Intercept & 86.104 & 1.315 & $65.478 *$ & 38.612 & 1.790 & $21.567^{*}$ \\
\hline Time & .005 & .007 & .816 & .073 & .013 & $5.417^{*}$ \\
\hline Assessment type & 4.014 & .765 & $5.244 *$ & -26.159 & 1.519 & $-17.217^{*}$ \\
\hline
\end{tabular}

Note: Assessment type $=$ random versus alcohol assessment. Control: $\mathrm{R}^{2}$ level $1=.03, \mathrm{R}^{2}$ level $2=.01$. Craving: $\mathrm{R}^{2}$ level $1=.06, \mathrm{R}^{2}$ level $2=.12$. * $\mathrm{P}$ value $<.001$.

\subsection{Perceived control, craving and alcohol consumption during alcohol assessments}

First, we investigated whether there was a difference in perceived control and craving between user-initiated alcohol assessments and random assessments. As mentioned above, both control and craving had significant variance on the second level (control: $\sigma_{\text {level } 2}^{2}=192.76$, $p<.001$, ICC $=.56$; craving: $\sigma_{\text {level } 2}^{2}=178.84, p<.001$, ICC $=.18$;), so these two research questions were tested with two multilevel analyses; one with control, and one with craving as the dependent variable (both located on level 1). In addition, both models included measurement occasion (time: used to account for the repeated measures) and an assessment type as level 1 predictors. Perceived control was significantly lower during alcohol assessments compared to random assessments $(b=5.244, t(1813)=4.21, p<.001$; see Table 3$)$. In addition, there was a significant difference in craving between random and user-initated alcohol assessments. That is, individuals had higher craving levels when they were drinking alcohol compared to the random assessments $(b=-26.16, t(2001)=-17.217 p<.001 ;$ see Table 3). Next, we looked at the relationships between control, craving and alcohol consumption within user-initated alcohol assessments only. In this subset of the data, no significant level 2 variance was found, so the relations between control, craving and alcohol consumption were investigated using multiple regression. We found, as hypothesized, that perceived control was negatively related to craving $(\beta=-.30$, $p<.001)$. Furthermore, we found that both increased craving and lower subjective self-control were associated with amount of alcoholic beverages (see Table 4).

\subsection{Perceived control as a moderator of the prospective relationship between craving and alcohol use}

We used PROCESS (Hayes, 2012) to test whether the relationship between increased craving and alcohol use during a user-initiated drinking session was stronger when an individual had a decreased control over alcohol use. The overall model was significant $\left(R^{2}=.29, F\right.$ $(3,388)=53.37, p<.001)$. Craving was positively related to the amount of beverages while a higher amount of perceived control was associated with less alcohol consumption (see Table 4). In addition, the moderator effect was significant $(b=-.001,95 \%$ CI [-.0013, -.0004], $t$ $=-3.70, p<.001)$. The interaction between craving and perceived

Table 4

Control and craving as predictors of anticipated alcohol consumption during a drinking session.

\begin{tabular}{lllll}
\hline & $\mathrm{b}$ & $\mathrm{SE}$ & $\mathrm{t}$ & $P$-value \\
\hline Constant & 19.653 & .160 & 126.227 & $p<.001$ \\
Craving & .0370 & .005 & 82.620 & $p<.001$ \\
Control & -.0374 & .009 & -4.405 & $p<.001$ \\
Craving x Control & -0.0009 & .002 & -3.698 & $p<.001$ \\
\hline
\end{tabular}

Note: $\mathrm{R}^{2}=.29$ 


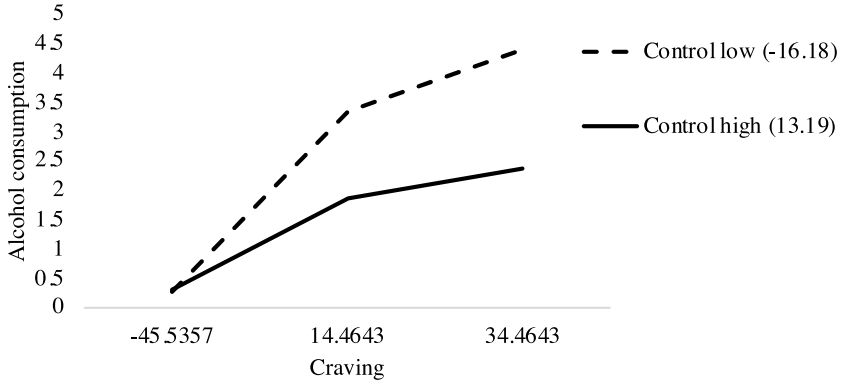

Fig. 1. Plot of the interaction effect between craving and subjective self-control on amount of alcohol consumed (standard drinks).

control led to a significant increase in variance predicted within the model $\left(R^{2}\right.$-change $\left.=.025, p<.001\right)$. As shown in the simple slope analysis in Fig. 1, the amount of alcoholic beverages participants consumed depended on both their perceived control and craving. When individuals reported lower levels of control, the amount of craving had more influence on alcohol consumption than when they perceived a high level of control. This finding supported our hypothesis that individuals with lower control over alcohol use have more problems controlling their drinking in response to craving.

\section{Discussion}

The aim of this study was to examine the predictive relationships between impaired control, craving and alcohol use in everyday life using EMA methods. For this purpose, we asked participants to initiate an EMA report of every drinking session they had in which planned alcohol consumption, perceived self-control and craving were assessed. In the meantime, we continued to assess amount of perceived control and craving in randomly prompted reports. If craving and impaired control are associated with alcohol use, one should expect periods of the day when control is low and craving is high to be followed by periods of increased drinking. In addition, we investigated whether selfcontrol was diminished when drinking alcohol and whether this is associated with the amount of alcohol participants consume. Finally, we tested whether craving is higher when drinking alcohol and whether this is predictive of amount of alcohol consumed. We further hypothesized that the relationship between craving and reported alcohol consumption would be moderated by reduced self-control over alcohol use.

First of all, results showed that impaired control was indeed prospectively related with the likelihood that people will drink. More specifically, on the measurements of self-control that preceded drinking (i.e., were within two hours of drinking) participants experienced less self-control compared to assessments that were not followed by drinking alcohol. The finding that control is associated with alcohol use is consistent with a large body of research that has demonstrated that control processes may be involved in drinking behaviour. For example, it has been shown that a poorer inhibitory control is associated with heavier alcohol use (Christiansen et al., 2012) and that manipulation of inhibitory control has a causal effect on alcohol consumption (Jones et al., 2011). Furthermore, this result is consistent with evidence that impaired control predicts problem drinking prospectively (Leeman et al., 2009). In addition, the Jones et al. (2018b) EMA study showed that while inhibitory control on a given day did not predicted daily alcohol consumption, change in inhibitory control across that day significantly predicted alcohol consumption later on that day. By demonstrating that control was lower prior to drinking alcohol in real-life drinking situations, the current study provides further support for the notion that impaired control is not merely an aspect of addiction, but may be involved in the development of problem drinking.

In addition to investigating the prospective relationships between control and alcohol use, we also examined impaired control in the field during drinking sessions. Results demonstrated that during (initial) drink consumption perceived control was lower than during random assessments. In addition, we found that during drinking sessions, control was related to amount of expected alcohol consumption. This could reflect the predictive relationship between self-control and drinking in which fluctuations in amount of control predict subsequent alcohol consumption. An alternative explanation could be that drinking causes decreased self-control. That is, the finding that control is lower during drinking is consistent with findings from previous studies that alcohol impairs inhibitory control (Field et al., 2010; Fillmore, 2003). For example, in a study by de Wit et al. (2000) it was found that alcohol affected the ability to inhibit responses. It is theorized that poor inhibitory control is related to drug use, both as a determinant and as a consequence (López-Caneda et al., 2014; Verdejo-Garcia et al., 2008; de Wit, 2009). The current findings may be in keeping with this notion. That is, while results show that control is lower on assessments that precede drinking compared to assessments that do not precede drinking, which is in line with the notion that control is linked to alcohol use as a determinant, it may well be the case that control further decreases as a consequence of drinking. In this way people may become trapped in a dynamic process in which lower self-control and alcohol consumption strengthen each other. However, because we only assessed planned drinks and control once at the start of the drinking sessions, we cannot determine whether drinking led to further changes in perceived control. We did, however, found that alcohol use within two hours before the random assessments was predictive of lower control and higher craving levels. It would be interesting to study the dynamic interplay between drinking and control by continuous or repeated measurement of these variables before, during and after drinking sessions in future studies.

Alcohol use was not only related to subjective self-control, but also with craving levels. That is, craving levels were higher on assessments that were within two hours of drinking alcohol, which gives support for the prospective relationship between craving and drinking. In addition, craving was higher on assessments when participants were drinking compared to other assessments. Futhermore, higher craving levels were associated with the amount of alcohol beverages participants were expected to drink during user-initiated alcohol assessments. These findings are well in line with findings from previous EMA studies that have shown a positive relationship between craving and substance use, both currently and prospectively (Serre et al., 2015; Shiffman et al., 2013). We also demonstrated that subjective self-control and craving assessed during the random assessments were significantly related. This relationship was stronger for assessments that were within two hours of drinking alcohol, although this effect was only marginally significant ( $p=.056)$. This builds on a number of previous demonstrations that self-reported impulsivity is related to craving (Evren et al., 2012; Joos et al., 2013).

Most importantly, findings indicated that subjective self-control acted as a moderator of the relationship between craving and alcohol consumption during drinking occasions. More specifically, temporary craving levels affected alcohol consumption to a higher extent in case someone reported lower levels of self-control over alcohol use. This finding is in line with the study of Wardell and colleagues (2015), demonstrating that high levels of trait impaired control in individuals moderated the within person associations of craving and alcohol use in the laboratory. Furthermore, the results of the current study are consistent with contemporary theories of addiction suggesting that both impairments in control processes and an increased motivation for substance use contribute to difficulties in controlling drug use (Goldstein and Volkow, 2002; Jentsch and Taylor, 1999; Wiers et al., 2007). This study adds weight to these theories by demonstrating the dynamic interplay between those important concepts in naturalistic settings, outside the laboratory. Studying this in real-time is important since self-control over alcohol use can be seen as a dynamic construct that is influenced by internal and environmental factors (Jones et al., 
2013). Further research is needed to investigate how self-control relates to other constructs in the natural environment such as alcohol-related cues (Jones and Field, 2015) and self-control demands (Muraven et al., 2005).

The present study benefitted from using a sample that consisted of participants from the general population regular drinkers which increases the generalizability of the findings. This is particularly important since impaired control is thought to be a first indicator of problem drinking. However, while half of the participants scored in the range of harmful alcohol use (Babor et al., 2001), it is not clear how the findings generalize to AUD patients. In addition, we did not examine intention or motivation to control alcohol consumption. It is thought that inhibitory control especially affects drinking if individuals are motivated to regulate their alcohol intake (Wiers et al., 2007). While the recruitment of a sample that did not necessarily state the intention to reduce drinking probably led to an underestimation of effects of reduced self-control on drinking, future research should include the motivation to reduce drinking. Related to that, we did not assess participants' intentions to initiate drinking. It may well be the case that when participants form the intention to drink alcohol, this result in subjective feelings of diminished self-control, increased craving levels as well as alcohol consumption. Although the potential role of intentions to drink on subjective sensations of diminished self-control and higher craving levels has not been empirically investigated, findings from a meta-analysis demonstrated that intentions to consume alcohol had a strong correlation with drinking (Cooke et al., 2016). In addition, in an EMA study by Jones et al. (2018b) daily intentions to drink alcohol significantly predicted alcohol consumption. Future studies should investigate whether diminished self-control and increased craving are associated with alcohol use, irrespective of intentions to consume or refrain from alcohol consumption.

A strong point of the study was that we examined the relationship between self-control over alcohol use and drinking as it unfolds over time. That is, we looked at measurements of self-control that preceded drinking episodes and in this way were able to study the prospective relationship. However, although the use of EMA reports allowed us to examine these important concepts in everyday life that cannot be obtained with other methodologies, the present study only suggests causal relations which should be confirmed by experimental studies. Associations can be the result of other variables and relations are likely to be bi-directional. In addition, while EMA methodology results in data that are more reliable and ecologically valid, it still suffers from limitations of self-report. This is especially important when assessing cognitive processes that people may not be completely aware of. Future studies combining more objective behavioral measures of self-control with the use of EMA will enhance the understanding of this important concept in real-life alcohol use. A small number of studies already successfully incorporated objective behavioral measures in EMA research studying cognitive processes in relation to alcohol use (Jones et al., 2018b), drug use (Marhe et al., 2013; Waters et al., 2012), snacking behavior (Powell et al., 2017), and smoking (Waters et al., 2013). Another limitation relates to the measurement of alcohol consumption. We asked participants to start an assessment when they were drinking and asked them to indicate the amount they thought they would drink in total. Therefore, we were not able to determine the actual amount of alcoholic drinks that were consumed during the drinking sessions. In addition, we have no information about the rate of planned alcohol intake since we did not stipulate a time period for planned alcohol consumption. In future studies this issue could be addressed by a follow up assessment after or while participants are drinking or ask them to start an assessment when they stop drinking. This is particularly relevant considering that participants lower in control are expected to drink more than planned. Another limitation that should be mentioned is the relatively low compliance rate of $47.4 \%$ on the random EMA assessments. A recent meta-analysis showed that the average compliance rate for EMA studies in the substance use field is $75.1 \%$ (Jones et al., 2019). In the current study, we did not reimburse participants which may have led to lower compliance rate. The findings from the meta-analysis, however, suggest that possible moderators such as reimbursement and assessment burden, do not influence compliance rate (Jones et al., 2019). To reduce the risk of noncompliance due to competing priorities, participants were allowed up to $90 \mathrm{~min}$ to complete the random assessments. However, allowing participants to delay their response poses the risk that we systematically missed data because participants may have delayed assessments at specific moments (e.g. in the company of others, at work or in class). Future research should investigate how to enhance compliance of EMA studies. Finally, further work is required to establish the environmental and internal events that are responsible for transient fluctuation in self-control and how these transient fluctuations relate to substance use behavior. For example, novel approaches to analyze EMA data focus on inter-individual variability and variability across time, which make it possible to understand the differences within an individual over time (Ginexi et al., 2014; Jongerling et al., 2015; Shiffman, 2013).

This is one of the first investigations in daily life of the relationship between real-time changes in self-control and alcohol consumption. Findings supported our hypotheses such that moments when subjective self-control was lower and craving levels were higher were followed by initiation of drinking. In addition, our results are consistent with predictions of theories of addiction that substance use might be affected by the interplay of control processes and an increased motivation to drink. These findings have several implications. First, it may be important to look at moment to moment fluctuations in control and craving as risk factors for drinking rather than only looking at general perceived control levels. Second, since drinking was lower at times when control was higher, it could be that control training is beneficial for reducing alcohol consumption in problem drinkers. Evidence supporting this idea comes from a meta-analysis that showed significant effects of inhibitory control training on alcohol consumption in the laboratory (Jones et al., 2016). However, results from a recent randomized controlled study outside the laboratory showed that internet delivered inhibitory control training had no specific effects on alcohol consumption in problem drinkers (Jones et al., 2018a). Future research should investigate how to improve inhibitory control training and examine whether this training is an effective intervention to reduce alcohol consumption in the real world. In addition, more research is needed to test whether the findings of the current study also apply to the clinical population of drinkers.

\section{Contributors}

Authors Remmerswaal and Franken conceived and designed the study and interpreted the findings. Author Remmerswaal wrote the first draft of the manuscript. Authors Jongerling and Remmerswaal conducted the statistical analyses. Author Eielts contributed to the analyses for this paper. Authors Franken, Jansen and Jongerling contributed to writing of this manuscript. All authors have reviewed and approved the final manuscript and the order of authors.

\section{Role of funding source}

Nothing declared.

\section{Declaration of Competing Interest}

The authors have no conflicts of interest to declare.

\section{Acknowledgements}

The authors thank Elske van den Broek, Jill van de Kasteele, Lindsay Heidstra, Merel Hillenaar, Nicolette Boddé, and Susan Ravensbergen for their contributions to the study. 


\section{Appendix A. Supplementary data}

Supplementary material related to this article can be found, in the online version, at doi:https://doi.org/10.1016/j.drugalcdep.2019.04. 043.

\section{References}

American Psychiatric Association, 2013. Diagnostic and Statistical Manual of Mental Disorders. Author. Washington, DC. Available from:. 5th ed. . https://www. psychiatry.org/.

Babor, T.F., Higgins-Biddle, J.C., Saunders, J.B., Monteiro, M.G., 2001. AUDIT: The alcohol use disorders identification test guidelines for use in primary care. Department of Mental Health and Substance Dependence, $2^{\text {nd }}$ ed. World Health Organization Available from: https://apps.who.int/iris/handle/10665/67205.

Baker, T.B., Piper, M.E., McCarthy, D.E., Majeskie, M.R., Fiore, M.C., 2004. Addiction motivation reformulated: an affective processing model of negative reinforcement. Psychol. Rev. 111, 33-51. https://doi.org/10.1037/0033-295X.111.1.33.

Baumeister, R.F., 2002. Ego depletion and self-control failure: an energy model of the self's executive function. Self Identity 1, 129-136. https://doi.org/10.1080/ 152988602317319302.

Baumeister, R.F., Vohs, K.D., Tice, D.M., 2007. The strength model of self-control. Curr. Dir. Psychol. Sci. 16, 351-355. https://doi.org/10.1111/j.1467-8721.2007.00534.x.

Bickel, W.K., Jarmolowicz, D.P., Mueller, E.T., Gatchalian, K.M., McClure, S.M., 2012. Are executive function and impulsivity antipodes? A conceptual reconstruction with special reference to addiction. Psychopharmacology 221, 361-387.

Christiansen, P., Cole, J.C., Goudie, A.J., Field, M., 2012. Components of behavioural impulsivity and automatic cue approach predict unique variance in hazardous drinking. Psychopharmacology 219, 501-510. https://doi.org/10.1007/s00213-0112396-z.

Cooke, R., Dahdah, M., Norman, P., French, D.P., 2016. How well does the theory of planned behaviour predict alcohol consumption? A systematic review and metaanalysis. Health Psychol. Rev. 10, 148-167. https://doi.org/10.1080/17437199. 2014.947547.

de Wit, H., 2009. Impulsivity as a determinant and consequence of drug use: a review of underlying processes. Addict. Biol. 14, 22-31. https://doi.org/10.1111/j.1369-1600. 2008.00129.x.

de Wit, H., Crean, J., Richards, J.B., 2000. Effects of d-amphetamine and ethanol on a measure of behavioral inhibition in humans. Behav. Neurosci. 114, 830-837.

Duckworth, A.L., Gendler, T.S., Gross, J.J., 2016. Situational strategies for self-control. Perspect. Psychol. Sci. 11, 35-55. https://doi.org/10.1177/1745691615623247.

Evren, C., Durkaya, M., Evren, B., Dalbudak, E., Cetin, R., 2012. Relationship of relapse with impulsivity, novelty seeking and craving in male alcohol-dependent inpatients. Drug Alcohol Rev. 31, 81-90. https://doi.org/10.1111/j.1465-3362.2011.00303.x.

Field, M., Wiers, R.W., Christiansen, P., Fillmore, M.T., Verster, J.C., 2010. Acute alcohol effects on inhibitory control and implicit cognition: implications for loss of control over drinking. Alcohol. Clin. Exp. Res. 34, 1346-1352. https://doi.org/10.1111/j. 1530-0277.2010.01218.x.

Fillmore, M.T., 2003. Drug abuse as a problem of impaired control: current approaches and findings. Behav. Cogn. Neurosci. Rev. 179-197. https://doi.org/10.1177/ 1534582303257007.

Fujita, K., 2011. On conceptualizing self-control as more than the effortful inhibition of impulses. Pers. Soc. Psychol. Rev. 15, 352-366. https://doi.org/10.1177/ 1088868311411165.

Ginexi, E.M., Riley, W., Atienza, A.A., Mabry, P.L., 2014. The promise of intensive longitudinal data capture for behavioral health research. Nicotine Tob. Res. 16, S73-S75. https://doi.org/10.1093/ntr/ntt273.

Goldstein, R.Z., Volkow, N.D., 2002. Drug addiction and its underlying neurobiological basis: neuroimaging evidence for the involvement of the frontal cortex. Am. J. Psychiatry 159, 1642-1652. https://doi.org/10.1176/appi.ajp.159.10.1642.

Hayes, A.F., 2012. PROCESS: A Versatile Computational Tool for Observed Variable Mediation, Moderation, and Conditional Process Modeling. Retrieved from. http:// www.afhayes.com/public/process2012.pdf.

Heather, N., Tebbutt, J.S., Mattick, R.P., Zamir, R., 1993. Development of a scale for measuring impaired control over alcohol consumption: a preliminary report. J. Stud. Alcohol 54, 700-709. https://doi.org/10.15288/jsa.1993.54.700.

Jellinek, E.M., 1960. The Disease Concept of Alcoholism. Hillhouse Press, New Haven, CT. https://doi.org/10.1037/14090-000.

Jentsch, J.D., Taylor, J.R., 1999. Impulsivity resulting from frontostriatal dysfunction in drug abuse: implications for the control of behavior by reward-related stimuli. Psychopharmacology 146, 373-390. https://doi.org/10.1007/PL00005483.

Jones, A., Christiansen, P., Nederkoorn, C., Houben, K., Field, M., 2013. Fluctuating disinhibition: implications for the understanding and treatment of alcohol and other substance use disorders. Front. Psychiatry 4, 1-10. https://doi.org/10.3389/fpsyt. 2013.00140.

Jones, A., Di Lemma, L.C., Robinson, E., Christiansen, P., Nolan, S., Tudur-Smith, C., Field, M., 2016. Inhibitory control training for appetitive behaviour change: a metaanalytic investigation of mechanisms of action and moderators of effectiveness. Appetite 97, 16-28. https://doi.org/10.1016/j.appet.2015.11.013.

Jones, A., Field, M., 2015. Alcohol-related and negatively valenced cues increase motor and oculomotor disinhibition in social drinkers. Exp. Clin. Psychopharmacol. 23, 122-129. https://doi.org/10.1037/pha0000011.

Jones, A., Guerrieri, R., Fernie, G., Cole, J., Goudie, A., Field, M., 2011. The effects of priming restrained versus disinhibited behaviour on alcohol-seeking in social drinkers. Drug Alcohol Depend. 113, 55-61. https://doi.org/10.1016/j.drugalcdep.2010. 07.006 .

Jones, A., McGrath, E., Robinson, E., Houben, K., Nederkoorn, C., Field, M., 2018a. A randomized controlled trial of inhibitory control training for the reduction of alcohol consumption in problem drinkers. J. Consult. Clin. Psychol. 86, 991-1004. https:// doi.org/10.1037/ccp0000312.

Jones, A., Remmerswaal, D., Verveer, I., Robinson, E., Franken, I.H., Wen, C.K.F., Field, M., 2019. Compliance with ecological momentary assessment protocols in substance users: a meta-analysis (in press). Addiction. https://doi.org/10.1111/add.14503.

Jones, A., Tiplady, B., Houben, K., Nederkoorn, C., Field, M., 2018b. Do daily fluctuations in inhibitory control predict alcohol consumption? An ecological momentary assessment study. Psychopharmacology 235, 1487-1496. https://doi.org/10.1007/ s00213-018-4860-5.

Jongerling, J., Laurenceau, J.P., Hamaker, E.L., 2015. A multilevel AR (1) model: allowing for inter-individual differences in trait-scores, inertia, and innovation variance. Multivariate Behav. Res. 50, 334-349. https://doi.org/10.1080/00273171. 2014.1003772.

Joos, L., Goudriaan, A.E., Schmaal, L., De Witte, N.A.J., Van den Brink, W., Sabbe, B.G.C., Dom, G., 2013. The relationship between impulsivity and craving in alcohol dependent patients. Psychopharmacology 226, 273-283. https://doi.org/10.1007/s00213012-2905-8.

Langenbucher, J.W., Chung, T., 1995. Onset and staging of DSM-IV alcohol dependence using mean age and survival-hazard methods. J. Abnorm. Psychol. 104, 346-354. https://doi.org/10.1037/0021-843X.104.2.346.

Leeman, R.F., Beseler, C.L., Helms, C.M., Patock-Peckham, J.A., Wakeling, V.A., Kahler, C.W., 2014. A brief, critical review of research on impaired control over alcohol use and suggestions for future studies. Alcohol. Clin. Exp. Res. 38, 301-308. https://doi. org/10.1111/acer.12269.

Leeman, R.F., Toll, B.A., Taylor, L.A., Volpicelli, J.R., 2009. Alcohol-induced disinhibition expectancies and impaired control as prospective predictors of problem drinking in undergraduates. Psychol. Addict. Behav. 23, 553-563. https://doi.org/10.1037/ a0017129.

Logan, G.D., Schachar, R.J., Tannock, R., 1997. Impulsivity and inhibitory control. Psychol. Sci. 8, 60-64. https://doi.org/10.1111/j.1467-9280.1997.tb00545.x.

López-Caneda, E., Rodríguez Holguín, S., Cadaveira, F., Corral, M., Doallo, S., 2014. Impact of alcohol use on inhibitory control (and vice versa) during adolescence and young adulthood: a review. Alcohol Alcohol. 49, 173-181. https://doi.org/10.1093/ alcalc/agt168.

Marhe, R., Waters, A.J., van de Wetering, B.J., Franken, I.H., 2013. Implicit and explicit drug-related cognitions during detoxification treatment are associated with drug relapse: an ecological momentary assessment study. J. Consult. Clin. Psychol. 81, 1-12. https://doi.org/10.1037/a0030754.

Miyake, A., Friedman, N.P., Emerson, M.J., Witzki, A.H., Howerter, A., Wager, T.D., 2000. The unity and diversity of executive functions and their contributions to complex "frontal lobe" tasks: a latent variable analysis. Cogn. Psychol. 41, 49-100. https://doi.org/10.1006/cogp.1999.0734.

Muraven, M., Collins, R.L., Shiffman, S., Paty, J.A., 2005. Daily fluctuations in self-control demands and alcohol intake. Psychol. Addict. Behav. 19, 140-147. https://doi.org/ 10.1037/0893-164X.19.2.140.

Papachristou, H., Nederkoorn, C., Havermans, R., van der Horst, M., Jansen, A., 2012. Can't stop the craving: the effect of impulsivity on cue-elicited craving for alcohol in heavy and light social drinkers. Psychopharmacology 219, 511-518. https://doi.org/ 10.1007/s00213-011-2240-5.

Powell, D.J., McMinn, D., Allan, J.L., 2017. Does real time variability in inhibitory control drive snacking behavior? An intensive longitudinal study. Health Psychol. 36, 356-364. https://doi.org/10.1037/hea0000471.

Rhemtulla, M., Fried, E.I., Aggen, S.H., Tuerlinckx, F., Kendler, K.S., Borsboom, D., 2016. Network analysis of substance abuse and dependence symptoms. Drug Alcohol Depend. 161, 230-237. https://doi.org/10.1016/j.drugalcdep.2016.02.005.

Robinson, T.E., Berridge, K.C., 2001. Incentive-sensitization and addiction. Addiction 96, 103-114. https://doi.org/10.1046/j.1360-0443.2001.9611038.x.

Schippers, G., Broekman, T., 2010. . De AUDIT. Nederlandse Vertaling Van De Alcohol Use Disorders Identification Test (Dutch Translation of the Alcohol Use Disorders Identification Test). Available from:. https://www.mateinfo.nl/audit/audit-nl.pdf.

Serre, F., Fatseas, M., Swendsen, J., Auriacombe, M., 2015. Ecological momentary assessment in the investigation of craving and substance use in daily life: a systematic review. Drug Alcohol Depend. 148, 1-20. https://doi.org/10.1016/j.drugalcdep. 2014.12.024.

Shiffman, S., 2009. Ecological momentary assessment (EMA) in studies of substance use. Psychol. Assess. 21, 486-497. https://doi.org/10.1037/a0017074.

Shiffman, S., 2013. Conceptualizing analyses of ecological momentary assessment data. Nicotine Tob. Res. 16, S76-S87. https://doi.org/10.1093/ntr/ntt195.

Shiffman, S., Dunbar, M., Kirchner, T., Li, X., Tindle, H., Anderson, S., Scholl, S., 2013. Smoker reactivity to cues: effects on craving and on smoking behavior. J. Abnorm. Psychol. 122, 264-280. https://doi.org/10.1037/a0028339.

Shiffman, S., Stone, A.A., Hufford, M.R., 2008. Ecological momentary assessment. Annu. Rev. Clin. Psychol. 4, 1-32. https://doi.org/10.1146/annurev.clinpsy.3.022806. 091415.

Verdejo-Garcia, A., Lawrence, A.J., Clark, L., 2008. Impulsivity as a vulnerability marker for substance-use disorders: review of findings from high-risk research, problem gamblers and genetic association studies. Neurosci. Biobehav. Rev. 32, 777-810. https://doi.org/10.1016/j.neubiorev. 2007.11.003.

Wardell, J.D., Ramchandani, V.A., Hendershot, C.S., 2015. A multilevel structural equation model of within-and between-person associations among subjective responses to alcohol, craving, and laboratory alcohol self-administration. J. Abnorm. 
Psychol. 124, 1050-1063. https://doi.org/10.1037/abn0000121.

Waters, A.J., Marhe, R., Franken, I.H., 2012. Attentional bias to drug cues is elevated before and during temptations to use heroin and cocaine. Psychopharmacology 219, 909-921. https://doi.org/10.1007/s00213-011-2424-z.

Waters, A.J., Szeto, E.H., Wetter, D.W., Cinciripini, P.M., Robinson, J.D., Li, Y., 2013. Cognition and craving during smoking cessation: an ecological momentary assessment study. Nicotine Tob. Res. 16, 111-118. https://doi.org/10.1093/ntr/ntt108.

Wiers, R.W., Bartholow, B.D., van den Wildenberg, E., Thush, C., Engels, R.C.M.E., Sher,
K.J., Grenard, J., Ames, S.L., Stacy, A.W., 2007. Automatic and controlled processes and the development of addictive behaviors in adolescents: a review and a model. Pharmacol. Biochem. Behav. 86, 263-283. https://doi.org/10.1016/j.pbb.2006.09. 021.

Wood, A.M., Kaptoge, S., Butterworth, A.S., Willeit, P., Warnakula, S., Bolton, T., et al., 2018. Risk thresholds for alcohol consumption: combined analysis of individualparticipant data for 599912 current drinkers in 83 prospective studies. Lancet 391, 1513-1523. https://doi.org/10.1016/S0140-6736(18)30134-X. 